\title{
Modelling and parameter identification of a semi-active vehicle damper
}

\author{
Michael Fleps-Dezasse Jakub Tobolář Johannes Pitzer \\ German Aerospace Center (DLR) \\ Institute of System Dynamics and Control \\ 82234 Wessling \\ Michael.Fleps-Dezasse@dlr.de Jakub.Tobolar@dlr.de Johannes.Pitzer@dlr.de
}

\begin{abstract}
In this paper two semi-physical models of the semiactive dampers of the DLR robotic electric vehicle ROboMObil (ROMO) are described and their implementation in Modelica is presented. Besides the damper characteristics and hysteresis, the models additionally consider the gas force and cover the differences of the damper characteristics for compression and rebound. A procedure to identify the damper model parameters was implemented using the DLR Optimization library. The measurement data used for parameter identification was recorded during experiments on a damper test bench. The simulation results of the damper models are compared to the experiment data of the semi-active damper and the suitability of the damper models with respect to accuracy and realtime simulation is discussed.
\end{abstract}

Keywords: semi-active damper; model identification, Bouc-Wen model, vehicle dynamics

\section{Introduction}

When designing a suspension system there are generally two conflicting goals concerning satisfactory ride comfort and good road-holding. Using passive dampers this leads to a compromise since good ride comfort can be achieved by a rather soft damping whereas for good road-holding high damping is necessary. On the contrary, suspension systems with controlled semi-active dampers can mitigate this restriction by allowing the adaption of the damper force according to the current vehicle state. A comprehensive overview of control strategies for semi-active suspensions is given in [1,2] or [3].

Semi-active dampers enable the continuous adaption of the damper force characteristics within a large operation range dependent on a control input. The adaption inside the damper is realized by modifying the force generating physical effects of the damper. Therefore, semi-active dampers generally need little energy effort for control as only small forces are necessary to modify the damping force [2]. These two properties, low energy consumption and continuous adaption of the damper force, make the integration of semi-active dampers in vehicle suspensions attractive. In order to investigate the influence of all the aforementioned aspects in the context of an experimental electric vehicle, semi-active dampers are used in the ROMO.

The ROMO, see Figure 1, is an innovative robotic electric vehicle developed at the Robotics and Mechatronics Center of the German Aerospace Center (DLR). It is composed of four Wheel Robots, see [4], which integrate drivetrain, brakes, steering, and suspension.

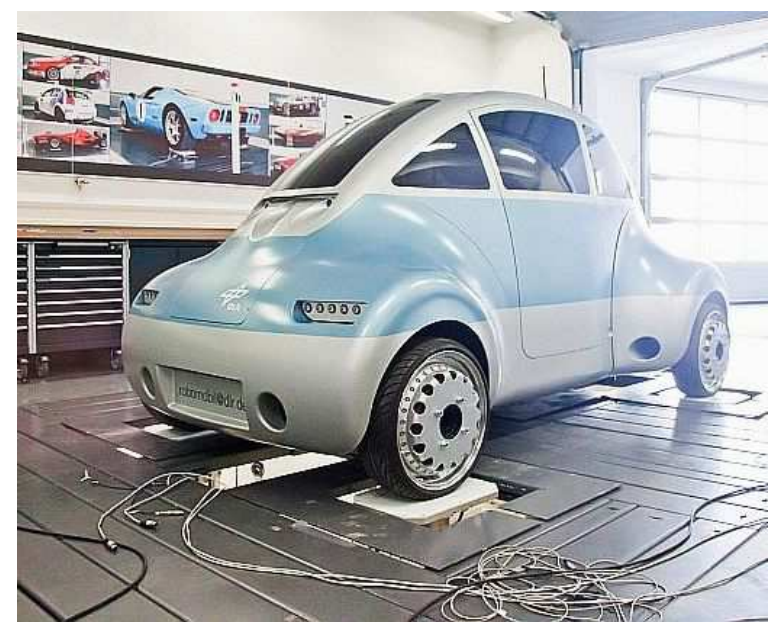

Figure 1: The ROboMObil (ROMO) on the four post test rig

During the design process of semi-active suspen- 
sions accurate purpose dependent models describing the behaviour of semi-active dampers are needed as shown e. g. in [5]. These models can then be used for full and quarter-car simulations as well as for estimator and controller design, see [6,7] or [1].

Due to the ability to combine models from different physical domains like mechanics and/or electrics with control algorithms the object oriented modelling language Modelica is well suited for modelling and simulation of controlled semi-active dampers. Another advantage for modelling of electric vehicles like the ROMO is the possibility to reflect the hierarchical model structure which simplifies the handling and parameterization of large models.

The common component models for semi-active dampers can be separated into the following groups depending on the kind of modelling:

- Full physical models which include a mechanical and an electrical model of the damper as well as a hydraulical model of the fluid flow.

- Semi-physical models which also include the damper mechanics and electrics, but approximate the hydraulics by an empirical model.

- Black-box models - empirical models which do not include physical model information in any way.

A description of a physical damper model can be found in $[8,9]$ or [10]. A good overview of semiphysical models can be found in [11] or [12]. These two latter papers give an introduction to semi-active damper modelling and describe several semi-physical models like the Bingham model or the Bouc-Wen model. A look-up table based damper model is developed in [13]. The application of a black-box damper model is shown in [14]. There, the authors develop a nonlinear autoregressive exogenous (ARX) model and compare it to an extended Bouc-Wen model according to [11]. The results presented in the paper illustrate that both models reproduce the damper behaviour with a high accuracy while the nonlinear ARX model slightly outperforms the extended Bouc-Wen model.

This paper focuses on two semi-physical damper models - a modified generalized extended Bouc-Wen model and a model based on a 2-dim. look-up table with the damper velocity and the control input as inputs, further referred to as Force Map based damper model. In contrast to the extended Bouc-Wen model presented by [11] and used in [14] and [2], the extended Bouc-Wen model described in this paper dis- tinguishes between compression and rebound (i. e. decompression) of the damper allowing different damper characteristics. This corresponds to the typical characteristics of semi-active dampers as used in vehicle suspension systems. Further, the dependency of the model parameters on the control input is modelled in a more general way, compared to [11], as the restriction imposed by the linear dependency of the parameters on the control input limits the performance of the extended Bouc-Wen model (see also [14]). Utilizing the implementation in Modelica, the model parameters are identified through a step by step optimization approach using the DLR Optimization library [15]. Hereby, the overall optimization task was split into several smaller subtasks each focusing on a subset of the overall model parameters and making use of particular experiment data specifically recorded for this optimization step. The advantage of this procedure is that the knowledge of the real damper structure and behaviour can be considered in the optimization algorithm and thus local minima finding can be avoided more easily during the optimization. Subsequently, the behaviour of the parameterized damper models is compared to the real damper behaviour and the feasibility of the damper models for real-time simulations is investigated.

The paper is structured as follows. In the next section the semi-active damper used in ROMO is presented. In section 3 the semi-active damper models are introduced and some implementation details are discussed whereas section 4 deals with the experimental setup and the identification approach. Subsequently, section 5 compares the simulated damper behaviour to the real damper behaviour.

\section{The semi-active damper}

In this work, a semi-active dual tube damper from the KW Automotive $\mathrm{GmbH}$ with one external controllable electromagnetic valve is used, see Figure 2. The adjustment of the damper force is realized by controlling the electric current flowing through the inductor of the electromagnetic valve. The induced magnetic field determines the position of the valve piston and consequently the oil flow through the valve. The flow direction of the oil through the external valve stays the same during both compression and rebound of the damper as the oil always flows from the rebound volume to the compensational volume.

Besides this electromagnetic valve there are further valves for compression and rebound, similarly to a conventional passive damper. They are placed at the 


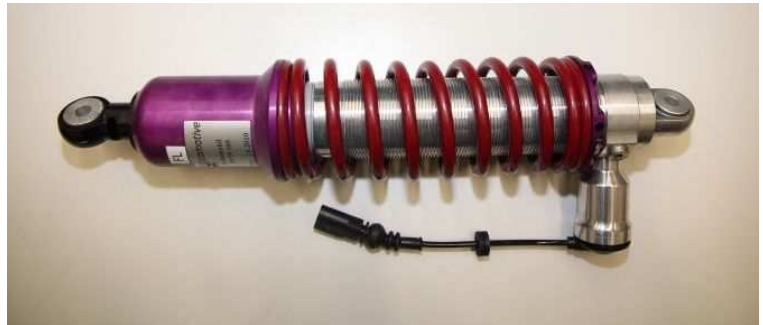

Figure 2: Semi-active dual tube damper with external continuously variable valve (coil spring was unmounted for experiments)

damper bottom and in the piston thus limiting the oil flow from the compression volume to the compensational volume and from the rebound volume to the compression volume, respectively.

Part of the compensational volume is filled with a pre-stressed gas to compensate the oil volume difference between pulled out damper and compressed damper caused by the piston rod. This gas volume can be regarded as an accumulator that generates a deflection dependent gas force. The gas force increases when compressing the damper and can threrefore be considered as a preloaded spring in a damper model.

The electric current to control the damper is generated by a power electric unit which transforms the Pulse-Width Modulated (PWM) signal, further referred to as control input, from the Rapid Control Prototyping (RCP) hardware into the appropriate electric current. Figure 3 illustrates the speed-force map of the damper at the constant control inputs $10 \%$ (blue) and 50\% (red) as recorded during experiments. The curves represent the raw damper force measurements and therefore reflect both the damper friction and the gas force influences.

The characteristic of the semi-active damper differs for compression (negative velocity) and rebound (positive velocity). This especially can be observed for larger control inputs as significantly higher rebound forces are generated, compared to compression ones, cf. Figure 3.

\section{Semi-active damper models}

The Force Map based damper model and the extended Bouc-Wen damper model were chosen for implementation in Modelica to investigate the achievable accuracy in reproducing the real damper behaviour and to evaluate their real-time feasibility.

In general, they both provide a good approximation of the highly non-linear behaviour of the semi-active

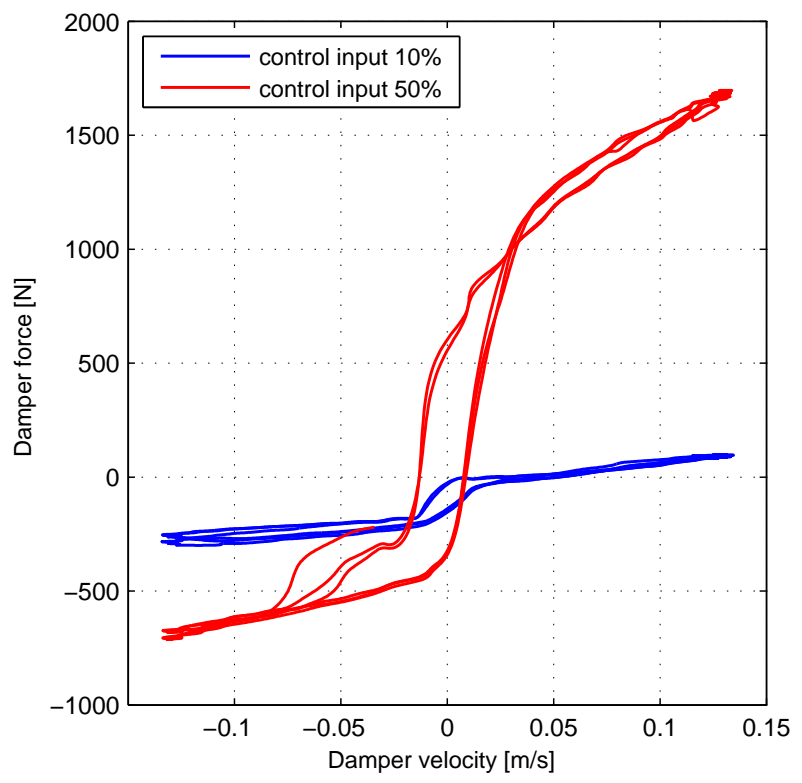

Figure 3: Speed-force map at control input $10 \%$ and $50 \%$

dampers, whereas the computational effort is far less than that of physical models as they do not include a model of the damper hydraulics, but approximate it by an empirical model. Therefore, these semi-physical models can be solved more easily by a fixed step solver and are better suited for real-time simulation, state estimation and controller design.

The damper models are implemented as onedimensional translational models and extend from the common translational interfaces of the Modelica Standard library.

\subsection{Generalized extended Bouc-Wen Model}

An introduction to the Bouc-Wen hysteres model can be found in [2]. The extended Bouc-Wen model as shown in Figure 4 is described in detail in [11]. There the authors started with a simpler model consisting of the linear damping element $c_{0}$, the linear spring $k_{0}$ and the Bouc-Wen hysteresis model. To better predict the real damper behaviour, they extended this simple model by the elements $c_{1}$ and $k_{1}$ which reproduce the gas chamber damping and the roll-off at low velocities, respectively. To make the model applicable for semi-active dampers they further determined a subset of three parameters out of the ten parameters of the extended Bouc-Wen model which are then linearly adapted according to the current control input. This way, the extended Bouc-Wen model is able to cover the different characteristics of semi-active dampers de- 
pending on the control input. Additionally, the control input was delayed by a first order low pass filter in order to better approximate the delayed real damper response towards changes in the control input due to the dynamic response of the electromagnetic valve and following the dynamic response of the damper hydraulics.

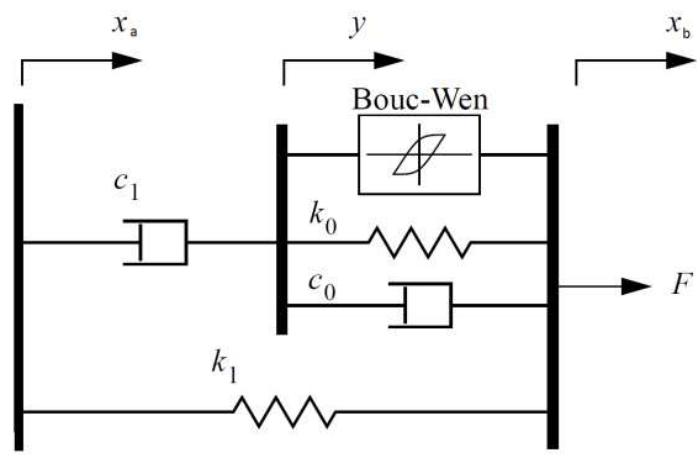

Figure 4: Mechanical model of the extended BoucWen damper model (similar to [11])

Based on the model description in [11], we further generalized the extended Bouc-Wen model as described in the following.

First, the set of three originally suggested parameters depending on the control input is extended to seven parameters and the linear dependency on the control input is replaced by a predefined look-up table, see structure of the implemented Modelica model in Figure 5. This way, the restrictions on the dependency between control input and model parameters are far less than in [11] and the damper model should achieve a better prediction of the real damper. Next, the parameters of the extended Bouc-Wen model are additionally modelled as a function of the current damper velocity to account for the different characteristics of the damper for compression and rebound, cf. Figure 3.

Furthermore, the low pass filter which approximates the dynamic damper response for changes in the control input is modelled as a first order transfer function (block firstOrder_varT) with an additional delay. The time constant as well as the delay time are considered to be functions of the instantaneous damper velocity (block relSpeed) and the sign of the derivative of the control input. This way, varying response times $T_{\text {comp }, r}, T_{\text {comp }, f}, T_{\text {rebound, } r}, T_{\text {rebound }, f}$ and delay times $\tau_{\text {comp }, r}, \tau_{\text {comp }, f}, \tau_{\text {rebound }, r}, \tau_{\text {rebound }, f}$ for compression and rebound as well as for rising and falling control inputs, indicated by the indices $r$ and $f$, respectively, can be considered.

Utilizing the abovementioned extensions, the result-

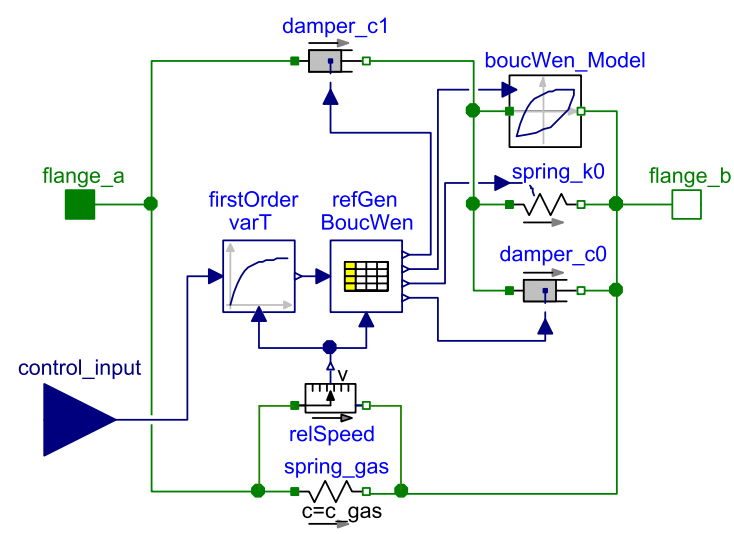

Figure 5: Generalized extended Bouc-Wen damper model

ing equations of the generalized extended Bouc-Wen model are given as

$$
\begin{aligned}
F_{d}= & c_{0}\left(u, v_{d}\right)\left(\dot{x}_{b}-\dot{y}\right)+k_{0}\left(u, v_{d}\right)\left(x_{b}-y\right) \\
& +k_{1}\left(\left(x_{b}-x_{a}\right)-x_{0}\right)+\alpha\left(u, v_{d}\right) z
\end{aligned}
$$

with

$$
\begin{aligned}
\dot{y}= & \frac{1}{c_{0}\left(u, v_{d}\right)+c_{1}\left(u, v_{d}\right)}\left(\alpha\left(u, v_{d}\right) z\right. \\
& +c_{0}\left(u, v_{d}\right) \dot{x}_{b}+c_{1}\left(u, v_{d}\right) \dot{x}_{a} \\
& \left.+k_{0}\left(u, v_{d}\right)\left(x_{b}-y\right)\right)
\end{aligned}
$$

and

$$
\begin{aligned}
\dot{z}= & -\gamma\left(u, v_{d}\right)\left|\dot{x}_{b}-\dot{y}\right| z|z|^{n-1} \\
& -\beta\left(u, v_{d}\right)\left(\dot{x}_{b}-\dot{y}\right)|z|^{n} \\
& +\delta\left(u, v_{d}\right)\left(\dot{x}_{b}-\dot{y}\right) .
\end{aligned}
$$

The meaning of the displacements $x_{a}, x_{b}$, which correspond to the motion of flange_a, flange_b, respectively, and the internal displacement $y$ is clear from Figures 4 and 5. The variable $z$ represents an internal state of the Bouc-Wen model and is often called hysteretic state. The spring stiffness $k_{1}$ and the unstretched spring length $x_{0}$ model the gas spring of the damper. Therefore, these parameters are considered invariant to changes of the control input $u$ and the damper velocity $v_{d}=\dot{x}_{b}-\dot{x}_{a}$. The remaining parameters of the generalized extended Bouc-Wen model are $k_{0}, c_{0}, \alpha, \beta, \gamma, \delta$ and $n$. All these parameters are functions of the control input $u$ and the damper velocity $v_{d}$ except for the exponent $n$, which is empirically determined to be $n=2$ as in [2].

It is assumed that the dependency of the parameters on the damper velocity $v_{d}$ can be approximated by a switching function which distinguishes between positive damper velocity for rebound and negative one for 
compression. This means that for every set $\theta_{e B W}$ of parameters two variants are generated - one for compression $\theta_{e B W, c o m p}$ and one for rebound $\theta_{e B W, \text { rebound }}$. These sets are then stored in the look-up table mentioned above. To combine the two sets of parameters the following smooth switching algorithm is applied:

$$
\theta_{e B W}=s \theta_{e B W, \text { rebound }}+(1-s) \theta_{e B W, c o m p},
$$

with the switching signal $s$ which is given by the following sigmoid function:

$$
s=0.5 \tanh \left(\frac{v_{d}}{v_{\text {eps }}}\right)+0.5 .
$$

This function generates a smooth output that changes from zero to one in a small region around zero damper velocities $v_{d}$. The size of this region is determined by $v_{\text {eps }}$. Although other switching signals like $s=\operatorname{sign}\left(v_{d}\right)$ would be possible, such a smooth switching signal is better suitable for a real-time simulations as no events occur. The function calculating the time constant of the first order transfer function and the delay time from the current damper velocity $v_{d}$ and control input derivative is implemented using a similar switching algorithm as the one described in equations (4) and (5).

\subsection{Force Map based model}

Figure 6 shows the Force Map based damper model implemented in Modelica. The control demand applied to the damper model is again (as in section 3.1) modelled by a transfer function and an additional delay with time constants and delay times dependent on the damper velocity $v_{d}$ and the control input derivative. The main component of this damper model is a lookUpTable, which takes the current damper velocity and the delayed control input as inputs and computes a force. Additionally, a model of the gas spring is included to improve the damper model for large deflections. The overall damper force is therefore given by:

$$
F_{d}=F_{\text {lookup }}+F_{\text {gas }},
$$

with the output of the look-up table $F_{\text {lookup }}$ and the gas force $F_{\text {gas }}$.

The implemented model is similar to the one developed in [13]. The main differences between these two models are the negligence of the damper housing stiffness which is very high and therefore causes stability problems during real-time simulations.

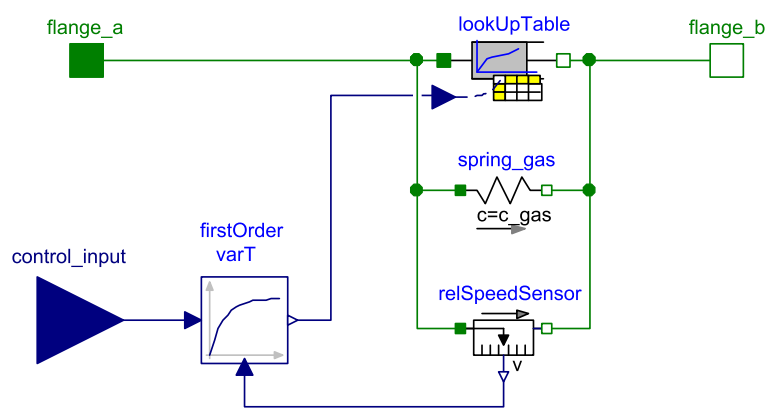

Figure 6: Force Map based damper model

\section{Parameter identification}

The parameter identification of the two semi-active damper models introduced in section 3.1 and 3.2 is a challenging task because both models are highly nonlinear and additionally the number of parameters to be determined is large. The arising optimization problem is non-convex and a lot of effort is necessary in order to avoid local minima evaluations. In this work, the optimization problem at hand is split into several subproblems as suggested in [13] and [2], estimating successively subsets $\theta_{j}$ of the overall parameter vector $\theta$.

The parameter subsets $\theta_{j}$ are chosen from experience and technical understanding of the damper in such a way that specific experiments focusing on identification of these parameters can be performed. The suboptimization problems are arranged in a chronological order and the estimated parameter subsets resulting from previous steps are inserted in the current optimization run. Although some of these suboptimization problems are non-convex as well, local minima can be avoided more easily by a careful choice of the initial values of the parameter subsets. This is achievable because the optimization problem is smaller compared to the overall optimization problem.

Throughout all suboptimizations, a cost function

$$
J=\frac{\frac{1}{N} \sum_{i=1}^{N}\left(F_{d, m}-F_{d, s}\left(\theta_{j}\right)\right)^{2}}{\frac{1}{N} \sum_{i=1}^{N}\left(F_{d, m}-\left(\frac{1}{T} \sum_{j=1}^{N} F_{d, m}\right)\right)^{2}}
$$

is used.

This cost function, which is also used by [14], represents the error-to-signal-ratio of the simulated damper force. Here, the number of the measured data samples is denoted by $N$. The measured damper force is represented by $F_{d, m}$ and the simulated damper force by $F_{d, s}$. 
The suboptimization problems can now be stated by

$$
\text { find } \underset{\theta_{j}}{\operatorname{argmin}}(J) \text { subject to } \theta_{j, \min }<\theta_{j}<\theta_{j, \text { max }} \text {, }
$$

with $\theta_{j, \max }$ and $\theta_{j, \min }$ restricting the maximum and minimum parameter values.

The optimization is done in Modelica/Dymola using the model optimization functions of the DLR Optimization library [15]. From the various optimization algorithms available in this library the Simplex method was chosen in this work as the algorithm proved satisfactory and fast convergence during preliminary investigations. A default cost function value larger than the initial cost function value was defined in the optimization setup to handle simulation runs with the generalized extended Bouc-Wen damper model which became unstable for certain parameter sets.

\subsection{Experimental Setup}

The experiments were performed on a Röhrig damper test rig which is shown in Figure 7.

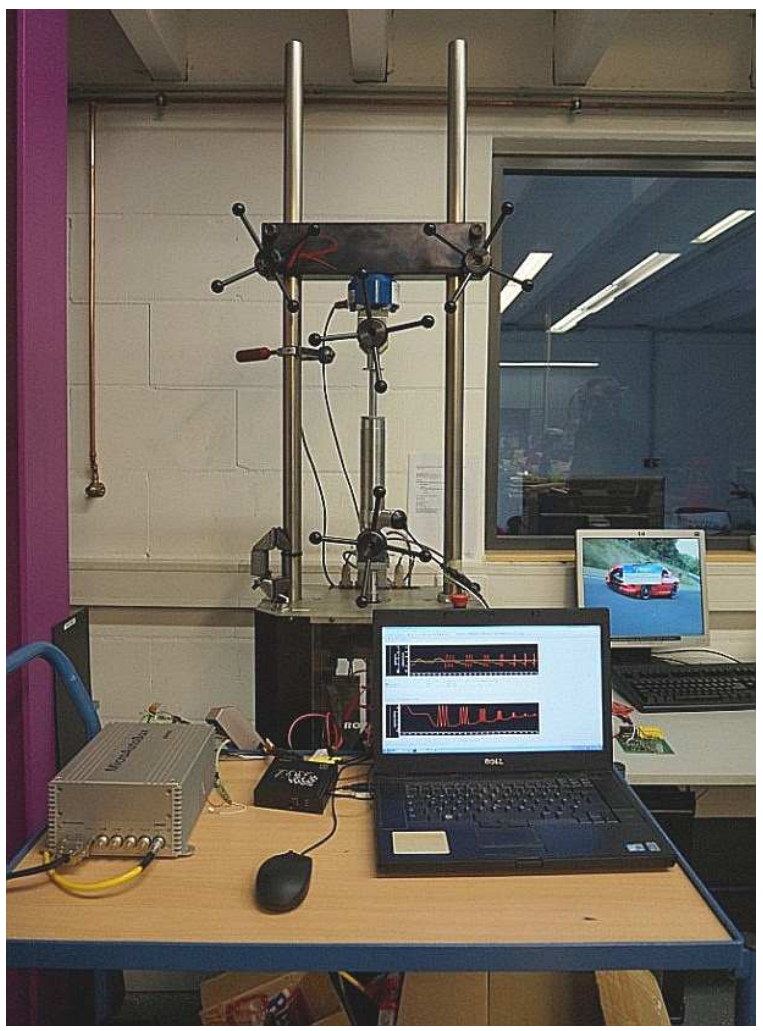

Figure 7: Experimental set-up

This test rig is equipped with a linear electric motor and therefore able to generate various damper excitations like sine sweeps or constant velocity periods. The measurement data was recorded using a Microautobox 2 from dSpace. The sample period of the RCP system was set to $T_{s}=1 \mathrm{~ms}$. The measured signals are damper force, displacement and acceleration. These signals are recorded together with the control input and are pre-filtered by a low-pass FIR filter.

\subsection{Step by Step optimization approach}

The size of the overall parameter vector $\theta_{e B W}$ of the generalized extended Bouc-Wen model depends on the number of nodes in the look-up table storing the control input dependent parameters as described in section 3.1. Each control input segment is represented by one row in this look-up table. The elements of the parameter vector $\theta_{e B W}$ are:

$$
\begin{aligned}
\theta_{e B W}= & \left(k_{0}, x_{0}, \theta_{e B W, i, 2}, \ldots, \theta_{e B W, i, m+1},\right. \\
& \left.T_{i, r}, T_{i, f}, \tau_{i, r}, \tau_{i, f}\right)^{T}
\end{aligned}
$$

with

$$
\theta_{e B W, i, j}=\left(c_{0, i, j}, k_{0, i, j}, c_{1, i, j}, \alpha_{i, j}, \beta_{i, j}, \gamma_{i, j}, \delta_{i, j}\right)^{T} .
$$

Here, $i$ stays for comp or rebound, $j=2, \ldots, m+1$ and $m$ is equal to the number of control input segments. This parameter vector is split into the following $m+2$ subsets.

- Gas force identification

$$
\theta_{e B W, 1}=\left(k_{0}, x_{0}\right)^{T}
$$

Experiment: Constant, very small velocity excitation for compression and rebound at constant minimum control input $u_{\min }$.

- Bouc-Wen parameter identification at constant control input $u_{1}$ :

$$
\theta_{e B W, j}=\left(\theta_{e B W, c o m p, j}, \theta_{e B W, \text { rebound }, j}\right)^{T} .
$$

Experiment: Sine excitation of the damper with an amplitude of $25 \mathrm{~mm}$ and a frequency of $0.8 \mathrm{~Hz}$.

- Time constant and delay estimation

$$
\begin{aligned}
\theta_{e B W, m+2}= & \left(T_{\text {comp }, r}, T_{\text {comp }, f}, T_{\text {rebound }, r},\right. \\
& T_{\text {rebound }, f}, \tau_{\text {comp }, r}, \tau_{\text {comp }, f}, \\
& \left.\tau_{\text {rebound }, r}, \tau_{\text {rebound }, f}\right)^{T} .
\end{aligned}
$$

Experiment: Saw tooth excitation of the damper with an amplitude of $25 \mathrm{~mm}$ and a constant damper velocity with an absolute value of $0.15 \mathrm{~ms}^{-1}$ for compression and rebound. 
As an example, Figure 8 shows the virtual test rig for the optimization of the Bouc-Wen parameters at control input $u_{1}$. Similar virtual test rigs are employed to optimize the other parameter subsets. Therefore, the measurements have to be replaced by the corresponding measurement data, e. g. with a different control input.

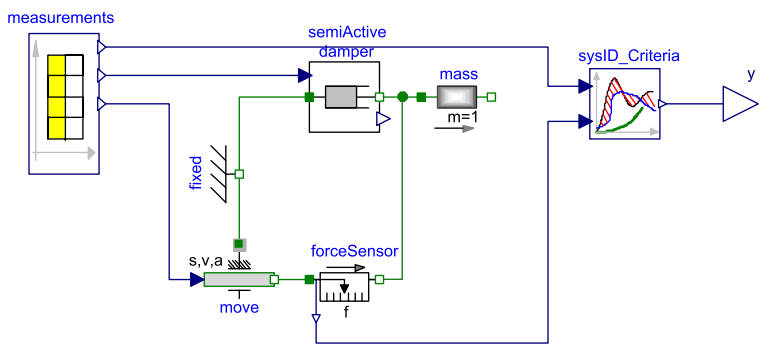

Figure 8: Virtual test rig for optimization

On the left-hand side of the model the measurement data is read by a CombiTimeTable called measurements, on the right-hand side the cost function is calculated by the sysID_Criteria block and in the middle the damper model semi-active damper is excited by a move element supplied with the measured displacement and derived velocity and acceleration.

The estimated gas force of step one is inserted as known value in the damper models for the following optimization steps and, further, the estimated parameters $\theta_{e B W, j}$ (with $j=2, \ldots, m+1$ ) of the previous step are used successively as initial values for the parameter vector $\theta_{e B W, j+1}$ of the following step. The initial values of the extended Bouc-Wen model for the first step are taken from [12] and the corresponding minimum parameter values $\theta_{j, \min }$ and maximum parameter values $\theta_{j, \max }$ are defined by scaling the initial parameter values.

The optimization procedure for the Force Map based damper model is very similar to the one of the extended Bouc-Wen model. The first step gas force estimation and the last step time constant estimation are the same. The steps in between are used to estimate the values of the lookUpTable, which takes the damper velocity and the control input as inputs and calculates a force as output. As the control input is constant during these steps, the values are again determined row by row.

\subsection{Optimization results}

The resulting cost function values for both damper models are shown in Table 1. The models reproduce the real damper behavior with almost the same accu- racy. For a control input of $10 \%$ the Force Map model and the extended Bouc-Wen model achieve the same accuracy. For a control input of 50\% the extended Bouc-Wen model achieves slightly better values than the Force Map model.

Table 1: Optimal cost function values of the damper models

\begin{tabular}{ccc}
\hline & \multicolumn{2}{c}{ Control input } \\
Damper model & $10 \%$ & $50 \%$ \\
\hline Extended Bouc-Wen & 0.097 & 0.090 \\
Force Map & 0.095 & 0.117 \\
\hline
\end{tabular}

The corresponding force over time, force over displacement and force over velocity diagrams for $10 \%$ and $50 \%$ control input are illustrated in the figures 9 and 10. The slight accuracy difference indicated by the cost function value for $50 \%$ control input is most obvious in the force over velocity diagram (Figure 10 below), mainly in the small velocity region.

In Figures 11 and 12 the results of the time constant and time delay identification for control input steps during rebound and compression are shown. The damper velocity was kept constant for rebound and compression to isolate the damper response for control input steps only. The modelled time behavior of the damper consisting of a first order transfer function and a delay with different time constants and delay times for rebound and compression as well as dependent on the direction of the control input change as described in section 3.1, is able to reproduce the damper behaviour very well. From Figures 11 and 12 it can further be seen that the delay is almost as large as the time constant of the transfer function and therefore the delay cannot be neglected by the damper model.

\subsection{Real-time suitability}

The parametrized damper models were simulated by a fourth-order Runge-Kutta solver with a fixed step size of $T_{s}=1 \mathrm{~ms}$ to analyse their feasibility for real-time simulations, as this solver is used to run the real-time model of the ROMO. The Force Map based damper model showed good results for this solver, while the generalized extended Bouc-Wen model became unstable for the optimized parameters. 

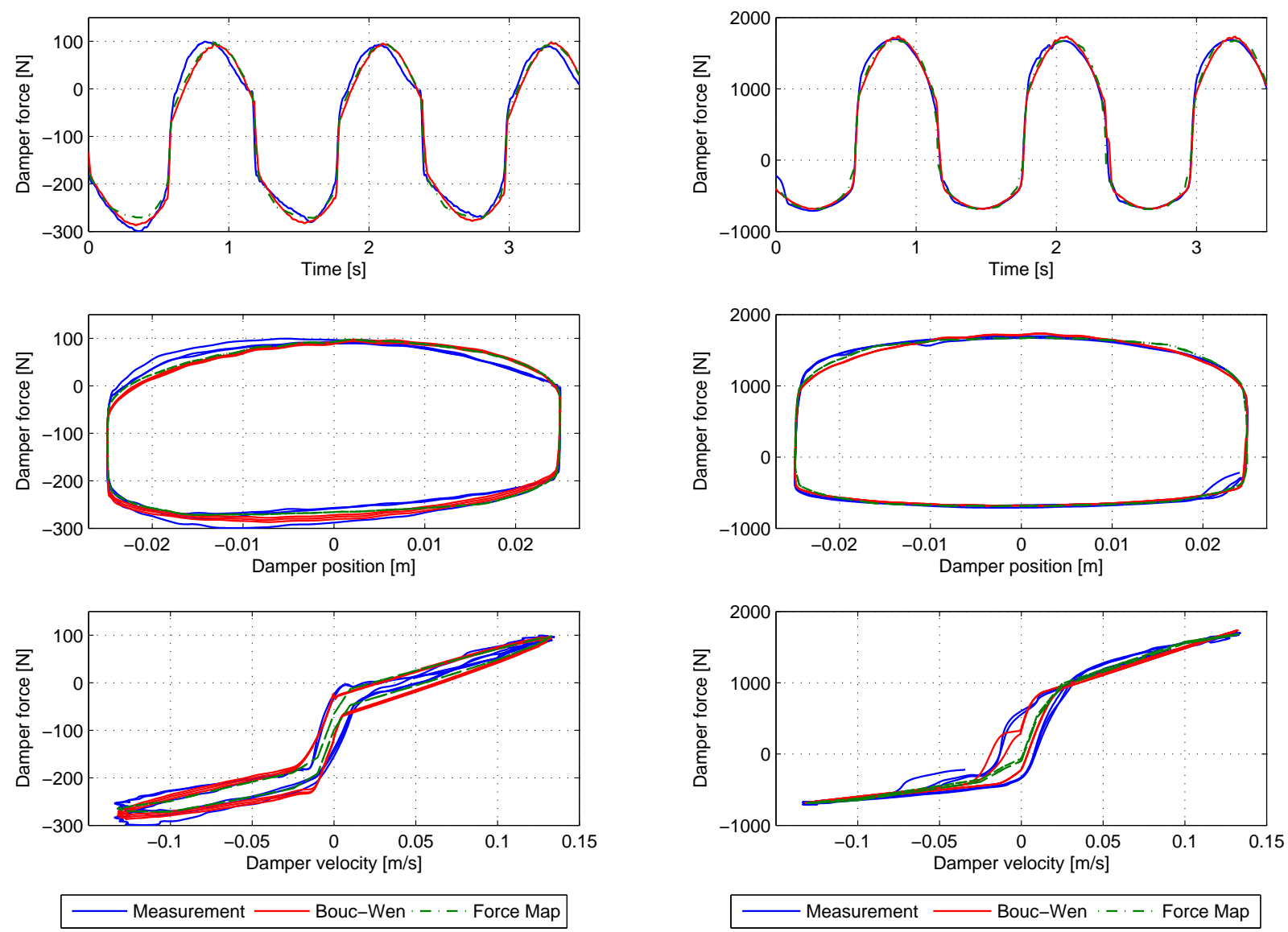

Figure 9: Comparison of damper models to damper measurements at $10 \%$ control input (above: force over time; middle: force over position; below: force over velocity)

Figure 10: Comparison of damper models to damper measurements at 50\% control input (above: force over time; middle: force over position; below: force over velocity)

\section{Conclusion}

In this paper two semi-physical damper models are presented and their accuracy in reproducing the real damper behavior is compared. Both models achieve a high accuracy in the considered velocity and frequency range. The generalized extended Bouc-Wen model gives more accurate results especially for higher control input.

On the other hand, the Force Map based damper model proved a better suitability for real-time simulation due to its more robust stability properties depending on the chosen solver and step-size and its simpler structure.

In the future, the velocity and frequency range of the damper models have to be extended towards higher values to improve the damper models for larger operation ranges. Further, the influence of stiction and sliding friction on the damper model accuracy for small

control inputs and small velocities have to be investigated as well.

\section{Acknowledgement}

The authors would like to thank Dr. Johann Bals and Mr. Jonathan Brembeck for their support and Mr. Uwe Bleck for his expertise in questions of applied vehicle dynamics. A special thank of the authors goes to Dr. Andreas Pfeiffer for his great support in application questions concerning the DLR Optimization library. The authors further would like to thank the KW Automotive GmbH for providing the semi-active damper and the damper test rig. 

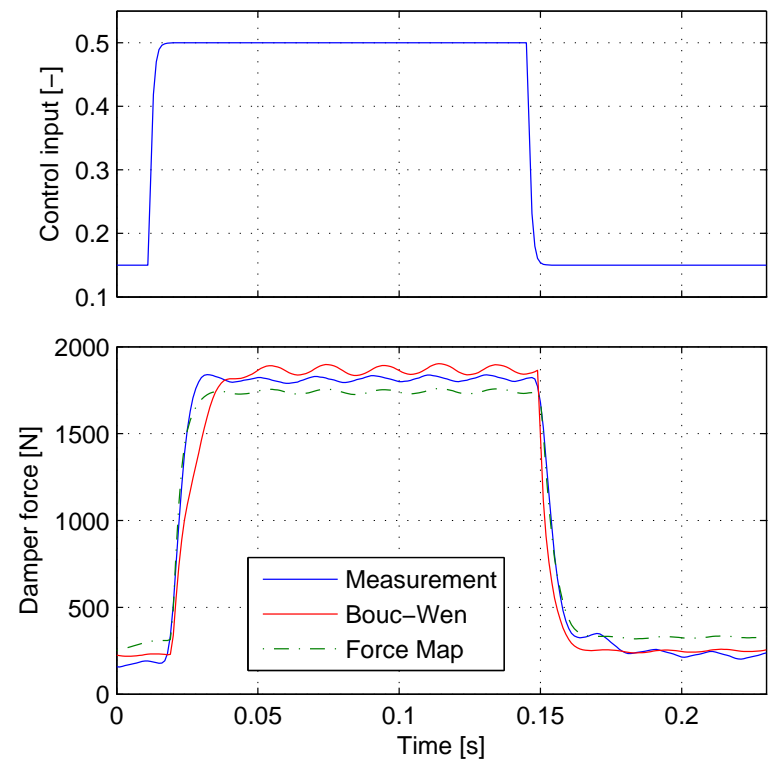

Figure 11: Force over time diagram with control input steps from $15 \%$ to $50 \%$ and back (during rebound)

\section{References}

[1] Savaresi S. M.: Semi-active suspension control design for vehicles. Amsterdam, Boston: Butterworth-Heinemann/Elsevier, 2010.

[2] Guglielmino E.: Semi-active suspension control: Improved vehicle ride and road friendliness. London: Springer, 2008.

[3] Valášek M. and Kortüm W.: EU-Project COPERNICUS: Development of Semi-Active RoadFriendly Truck Suspension: Choice of Control Law, Experimental Verification, Implementation, Results. Aachen, 2000.

[4] Brembeck J., Ho L. M., Schaub A., C. Satzger, Tobolar J., Bals J. and Hirzinger G.: ROMO - the robotic electric vehicle. In: International Association for Vehicle System Dynamics (IAVSD), 2011.

[5] Kortüm W., Valášek M., Šika Z., Schwartz W., Steinbauer P., and Vaculín O.: Semi-active damping in automotive systems: Design-bysimulation. International Journal of Vehicle Design, vol. 28, no. 1, pp. 103-120, 2002.

[6] Fleps-Dezasse M. and Brembeck J.: Model Based Vertical Dynamics Estimation with Modelica and FMI. In: Advances in Automotive Control, Elsevier, IFAC, pp. 341-346, 2013.

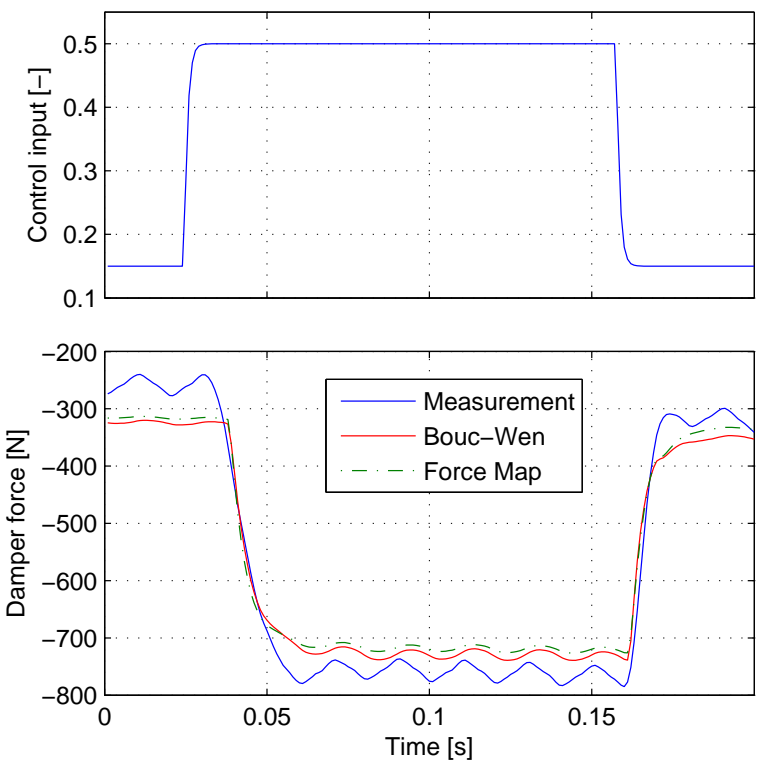

Figure 12: Force over time diagram with control input steps from $15 \%$ to $50 \%$ and back (during compression)

[7] Koch G., Kloiber T. and Lohmann B.: Nonlinear and filter based estimation for vehicle suspension control. In: 49th IEEE Conference on Decision and Control (CDC), pp. 5592-5597, 2010.

[8] Duym S. W., Stiens R., and Reybrouk K.: Evaluation of Shock Absorber Models. Vehicle System Dynamics, vol. 27, no. 2, pp. 109-127, 1997.

[9] Duym S. W.: Simulation Tools, Modelling and Identification, for an Automotive Shock Absorber in the Context of Vehicle Dynamics. Vehicle System Dynamics, vol. 33, no. 4, pp. 261285, 2000.

[10] Pellegrini E., Koch G., and Lohmann B.: Physical Modeling of a Nonlinear Semi-Active Vehicle Damper. In: Advances in Automotive Control: IFAC, Elsevier, pp. 324-329, 2010.

[11] Spencer Jr. B. F., Dyke S. J., Sain M. K. and Carlson J. D.: Phenomenological Model for Magnetorheological Dampers. J. Eng. Mech, vol. 123, no. 3, pp. 230-238, 1997.

[12] Butz T. and von Stryk O.: Modelling and Simulation of Electro- and Magnetorheological Fluid Dampers. ZAMM - Journal of Applied Mathematics and Mechanics, vol. 82, no. 1, pp. 320, 2002. 
[13] Altmann F.: Identifizierung eines magnetorheologischen Dämpfers: Vorbereitung des Modells eines leichten individuellen Stadt-Autos (LISA) zur Simulation unter Verwendung des semiaktiven Dämpfers. Diploma thesis, 2004.

[14] Savaresi S. M., Bittanti S. and Montiglio M.: Identification of semi-physical and black-box non-linear models: the case of MR-dampers for vehicles control. Automatica, vol. 41, no. 1, pp. 113-127, 2005.

[15] Pfeiffer A.: Optimization library for interactive multi-criteria optimization tasks. In: Modelica 2012 Conference, 2012. 